\title{
Streptococcal Infection-related Nephritis (SIRN) Manifesting Membranoproliferative Glomerulonephritis Type I
}

\author{
Ken Iseri ${ }^{1}$, Masayuki Iyoda ${ }^{1}$, Yasutaka Yamamoto ${ }^{1}$, Naoto Kobayashi ${ }^{1}$, Takashi Oda ${ }^{2}$, \\ Yutaka Yamaguchi ${ }^{3}$ and Takanori Shibata ${ }^{1}$
}

\begin{abstract}
We herein report the case of an 18-year-old boy who developed nephrotic syndrome and hypertension after upper airway inflammation. Post-streptococcal acute glomerulonephritis was diagnosed on the basis of a high antistreptolysin $\mathrm{O}$ titer, hypocomplementemia, proteinuria, and microscopic hematuria. A renal biopsy was performed due to persistent proteinuria, and the pathological diagnosis was membranoproliferative glomerulonephritis (MPGN) type I. Glomeruli showed positive staining for nephritis-associated plasmin receptor (NAPlr), a nephritogenic group A streptococcal antigen, and plasmin activity was found in a similar distribution as NAPlr deposition. This rare case of streptococcal infection-related nephritis (SIRN) manifesting MPGN type I supports the histological diversity of SIRN.
\end{abstract}

Key words: membranoproliferative glomerulonephritis (MPGN), nephritis-associated plasmin receptor (NAPlr), post-streptococcal acute glomerulonephritis (PSAGN), streptococcal infection-related nephritis (SIRN), nephrotic syndrome

(Intern Med 55: 647-650, 2016)

(DOI: 10.2169/internalmedicine.55.5409)

\section{Introduction}

The renal pathological findings associated with poststreptococcal acute glomerulonephritis (PSAGN) include diffuse endocapillary proliferative glomerulonephritis (GN), granular deposition of C3 with or without immunoglobulin (Ig)G, and subepithelial deposits referred to as "humps." Yoshizawa et al. isolated and characterized a nephritogenic antigen from group A streptococcus (GAS) that they named "nephritis-associated plasmin receptor" (NAPlr) $(1,2)$. NAPlr is homologous to the streptococcus plasmin receptor known as streptococcal glyceraldehyde-3-phosphate dehydrogenase (GAPDH) and causes glomerular damage in PSAGN by trapping plasmin and maintaining its activity (3). The detection of NAPlr and plasmin activity in glomeruli is thus useful for diagnosing GN induced by a streptococcal infection. Recent observations have revealed that NAPlr deposition and plasmin activity can be found in some cases of other glomerular diseases, such as Henoch-Schönlein pur- pura nephritis (4) and C3 glomerulopathy (5-9). Oda et al. referred to these diseases (GN induced by streptococcal infection and subsequent glomerular deposition of NAPlr and plasmin activity) collectively as streptococcal infectionrelated nephritis (SIRN) (10).

We herein report the case of a patient with SIRN who developed nephrotic syndrome (NS). A renal biopsy showed membranoproliferative glomerulonephritis (MPGN) type I accompanied by the deposition of NAPlr. To the best of our knowledge, only a small number of reports have described MPGN type I as a renal pathological finding of $\operatorname{SIRN}(7,8)$. We herein discuss the pathogenesis of this case.

\section{Case Report}

A healthy 18-year-old boy developed cold-like symptoms that resolved without taking medicine. However, systemic edema with weight gain (+7 kg in 3 days) appeared approximately 2 weeks after the onset of symptoms, and he was admitted to our hospital for an examination and treatment. On

${ }^{1}$ Division of Nephrology, Department of Medicine, Showa University School of Medicine, Japan, ${ }^{2}$ Renal Unit of Internal Medicine, Hachioji Medical Center, Tokyo Medical University, Japan and ${ }^{3}$ Yamaguchi's Pathology Laboratory, Japan

Received for publication March 23, 2015; Accepted for publication June 16, 2015

Correspondence to Dr. Masayuki Iyoda, iyoda@med.showa-u.ac.jp 

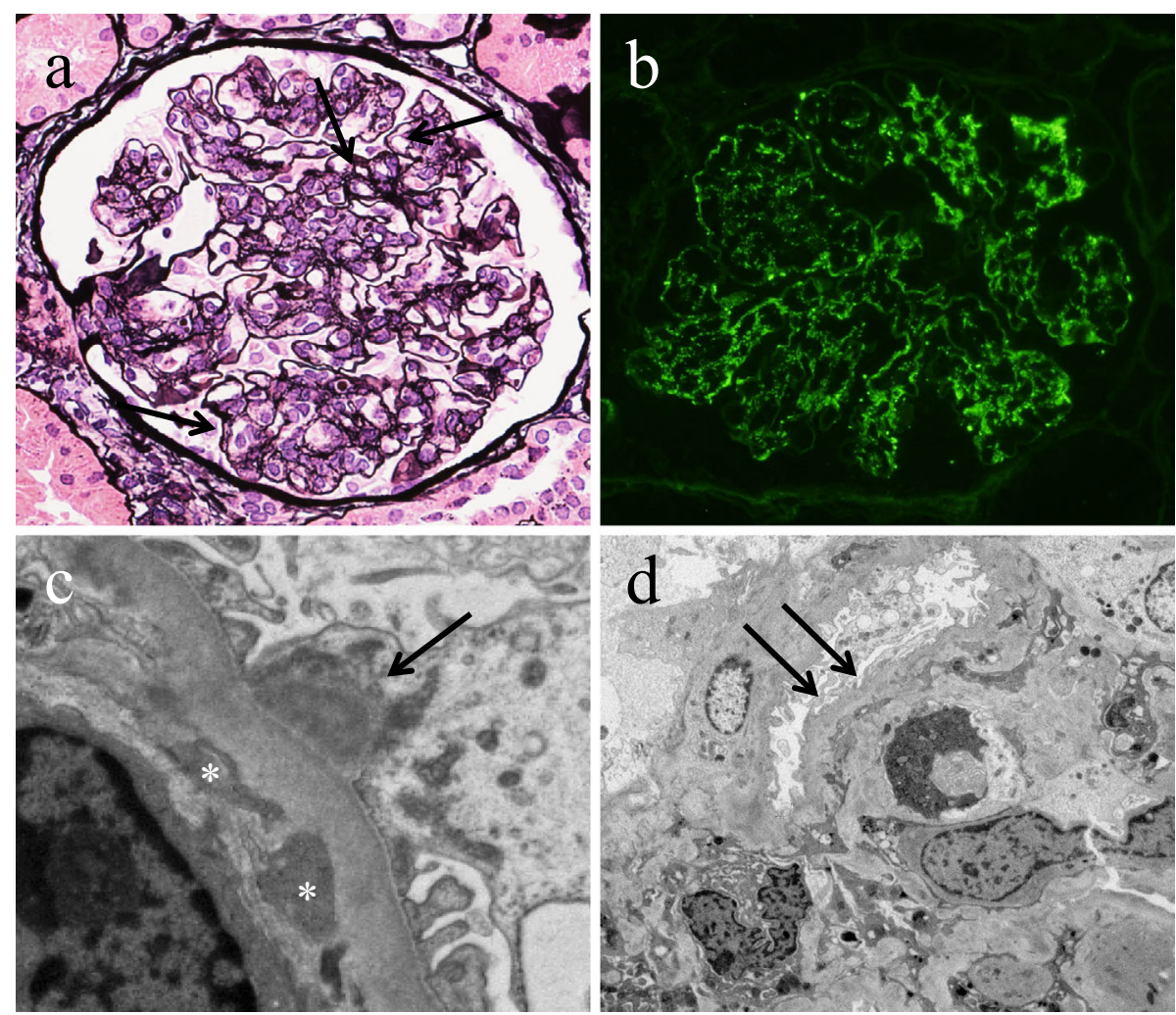

Figure 1. Kidney biopsy findings. a) Light microscopy findings in a periodic acid-silver methenamine (PAM)-stained paraffin-embedded section. Thick glomerular basement membranes (GBMs) and double contours of the GBMs were seen (arrows). b) Immunofluorescence shows granular staining for IgG in the mesangial area and capillary walls. c) An electron micrograph shows marked subendothelial electron-dense deposits (asterisks). Hump-shaped subepithelial deposits (arrow) are seen. d) Mesangial interposition is apparent (arrows).

physical examination, his temperature was $36.6^{\circ} \mathrm{C}$ and blood pressure was $158 / 76 \mathrm{mmHg}$. His height was $174 \mathrm{~cm}$, and his weight was $68.9 \mathrm{~kg}$. Facial and lower extremity edema was evident. He had no family history of kidney disease. The following laboratory results were obtained: albumin (Alb), $3.0 \mathrm{~g} / \mathrm{dL}$ (normal, 4.0-5.1 g/dL); blood urea nitrogen (BUN), $44.0 \mathrm{mg} / \mathrm{dL}$ (normal, 3.8-8.0 $\mathrm{mg} / \mathrm{dL}$ ); creatinine $(\mathrm{Cr}), 1.27 \mathrm{mg} / \mathrm{dL}$ (normal, 0.6-1.2 mg/dL); complement $\mathrm{C} 3$ $12.6 \mathrm{mg} / \mathrm{dL}$ (normal, $80-160 \mathrm{mg} / \mathrm{dL}$ ); C4 $10.0 \mathrm{mg} / \mathrm{dL}$ (normal, $10-40 \mathrm{mg} / \mathrm{dL}$ ); CH50 $7 \mathrm{U} / \mathrm{mL}$ (normal, $30-45 \mathrm{U} / \mathrm{mL}$ ); antistreptolysin O (ASO) titer 1,180 IU/mL (normal, $\leq 408$ $\mathrm{IU} / \mathrm{mL}$ ); and antistreptokinase (ASK) titer 2,560 (normal, $\leq 1,280$ ). Cryoglobulin and antinuclear antibody were undetectable. On a urinalysis, the sediment contained 30-49 red blood cells (RBCs)/high-power field (HPF). The 24-hour urinary protein excretion was $2.4 \mathrm{~g} /$ day. The fractional excretion of sodium (FENa) was $0.05 \%$.

Although the patient's blood pressure and systemic edema were attenuated by conventional therapy comprising sodium restriction, a calcium channel blocker, and diuretics, he developed NS (proteinuria, $3.87 \mathrm{~g} /$ day) and microscopic hematuria persisted.

A percutaneous kidney biopsy was performed on hospital day 14 to determine the cause of persistent NS. Light microscopy (LM) sections contained 11 glomeruli with no sclerosis or crescents, while diffuse mesangial hypercellularity, endocapillary proliferation with inflammatory cell infiltration (mostly neutrophils), thick glomerular basement membranes (GBMs), and double contours of the GBMs were observed (Fig. 1a). Immunofluorescence microscopy (IF) showed intense staining of IgG (Fig. 1b) and C3 in the mesangial area and capillary walls. Electron microscopy (EM) showed mesangial and subendothelial electron-dense deposits (EDD) (Fig. 1c) and mesangial interposition (Fig. 1d). A glomerular capillary containing isolated small hump-shaped, subepithelial deposits was observed (Fig. 1c). No organized structures were found in EDD. Positive staining for NAPlr was seen in the glomeruli (Fig. 2a) and staining for plasmin activity by in situ zymography matched the area staining for NAPlr (Fig. 2b). On the other hand, C3 staining was located in a different area from NAPlr staining (Fig. 2c, d). According to these histological findings, we diagnosed the patient with MPGN type I associated with group A streptococcal infection.

Although we planned to start prednisolone therapy in consideration of MPGN with nephrotic-range proteinuria, the patient's proteinuria gradually improved after the renal biopsy and he spontaneously achieved complete remission. He was discharged in a good general condition on hospital day 25. At that time, the test results showed: Alb, $3.9 \mathrm{~g} / \mathrm{dL}$; $\mathrm{Cr}$, 

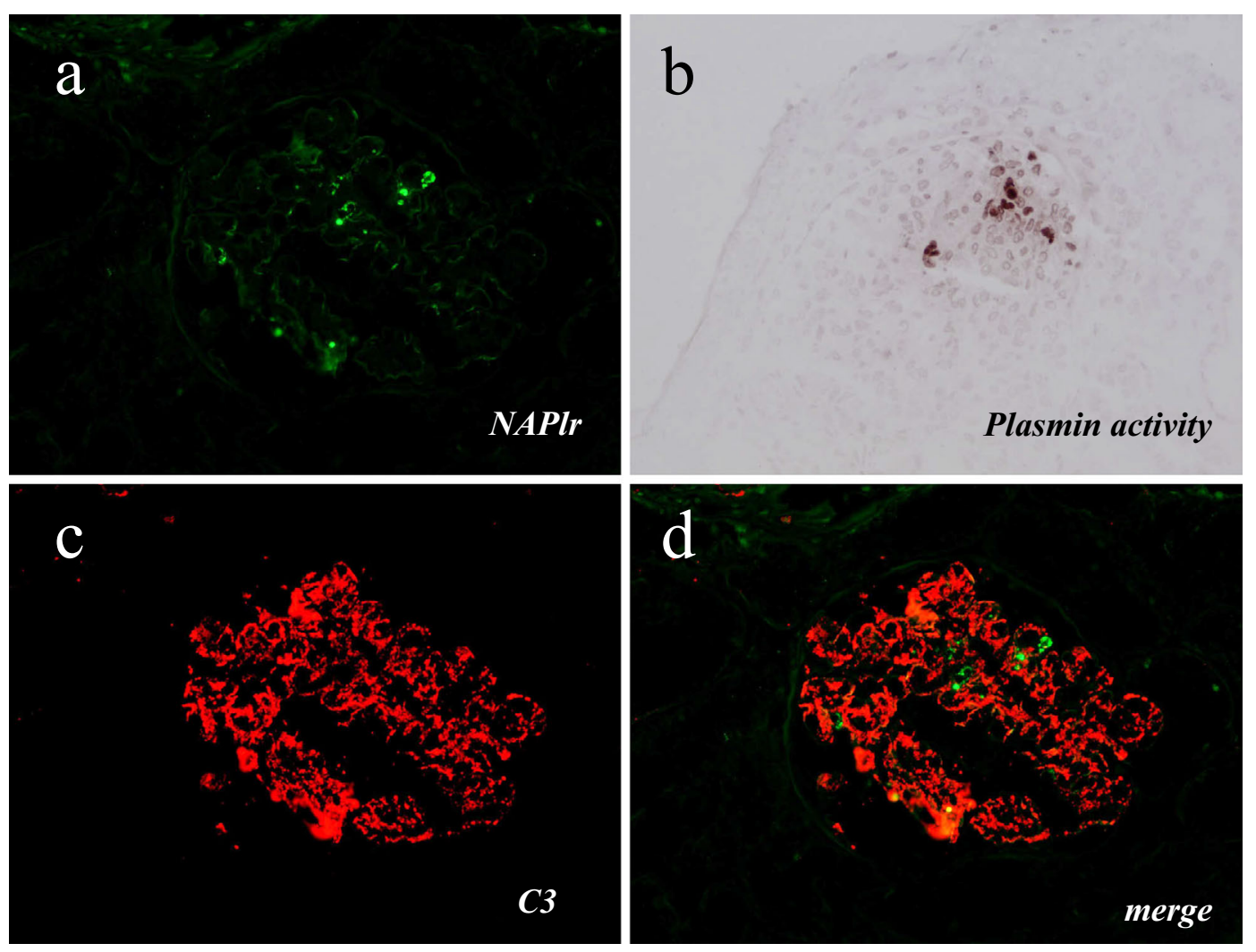

Figure 2. Immunofluorescent study. a) Positive staining for NAPIr is seen in the glomeruli. b) Plasmin activity by in situ zymography is found in the same area as NAPlr staining. c) Intense C3 staining in the mesangial area and capillary walls. d) A merged image showing the different distributions of NAPlr and C3 deposition.

$0.83 \mathrm{mg} / \mathrm{dL} ; \mathrm{C} 3,28.8 \mathrm{mg} / \mathrm{dL}$; C4, $24.1 \mathrm{mg} / \mathrm{dL}$; and CH50, $20 \mathrm{U} / \mathrm{mL}$. A urinalysis showed weakly positive results for protein and the sediment contained 10-19 RBCs/HPF. The complement levels and microscopic hematuria normalized 2 months after discharge.

\section{Discussion}

In the present case, the patient developed NS two weeks after the onset of cold-like symptoms. The renal biopsy revealed diffuse mesangial and endocapillary proliferation with double contour of the GBMs on LM, showing an MPGN-like lesion. IF showed intense staining of IgG and $\mathrm{C} 3$ in the mesangium and capillary walls. EM showed mesangial and subendothelial EDD, accompanied by mesangial interposition and hump-shaped subepithelial deposits. SIRN was suggested according to the elevated ASO titer, complete remission with only supportive therapy, endocapillary proliferation with hump-shaped subepithelial deposits, and the NAPlr deposition and plasmin activity observed in the glomeruli. SIRN in the present case was characterized by NS and MPGN. Lupus nephritis or cryoglobulinemia were clinically ruled out, and complete spontaneous remission made the diagnosis of idiopathic MPGN unlikely in this case.

Although it is difficult to distinguish between typical
PSAGN manifesting endocapillary proliferative GN and early stage MPGN with leucocytes infiltration within the capillary lumen, reported cases of typical MPGN type I with double contours of the GBMs and marked subendothelial electron-dense deposits associated with a streptococcal infection confirmed by immunostaining for NAPlr and plasmin are limited. Hump-shaped subepithelial deposits were found in this case which provided additional evidence of streptococcal infection. Accordingly, we diagnosed this case as SIRN manifesting MPGN type I. It has been reported that SIRN exhibits diverse histologically findings according to the disease, such as in Henoch-Schönlein purpura nephritis (4) and C3 glomerulopathy (5-9). Uchida et al. reported a case of PSAGN with massive subendothelial deposits, but no subepithelial deposits, histologically resembling C3 glomerulopathy (8). They speculated that streptococcal skin infections, such as in their case, might preferentially induce subendothelial deposition in consideration of previous reports that showed that 7 of 27 patients with PSAGN associated with streptococcal pyoderma displayed subendothelial deposits (11). In addition, cases of dense deposit disease (DDD) associated with a group A streptococcus infection have been reported $(4,5,10,12)$. Interestingly, the disease resolved in most of these patients with minimal treatment without the progression to end-stage renal failure despite persistently low serum C3 concentrations. 
NAPlr is known as a potential cause of PSAGN and is capable of activating the alternate pathway of the complement system (13). Because NAPlr is co-localized with plasmin, but not with complement or IgG as shown in the present case, nephritogenicity was considered to be related to the plasmin-binding capacity, facilitating immune complex deposition and subsequent inflammation (3). Suga et al. reported a case of DDD associated with a group A streptococcal infection without the involvement of $\mathrm{C} 3 \mathrm{NeF}$ or $\mathrm{CFH}$ deficiency. In their case, NAPlr and plasmin activity in the glomeruli persisted 6 months after disease onset, which generally disappears within one month (5). They speculated that the persistent deposition of NAPlr might play a role in the development of DDD through the continuous trapping of immune complexes accompanied by complement activation. This clinical situation appears plausible as a pathogenesis for MPGN formation with persistent urinary abnormalities in the present case. However, the underlying causes of persistent deposition of NAPlr remain unclear.

In conclusion, we reported a rare case of SIRN histologically manifesting MPGN type I. Although the definitive explanation for the pathogenesis of MPGN type I formation in the present case remains unclear, we speculate that the continuous activation of the alternate complement pathway, caused by persistent NAPlr deposition, is one of the critical factors in this disease. Our case supports the histological diversity of SIRN.

The authors state that they have no Conflict of Interest (COI).

\section{References}

1. Yoshizawa N, Yamakami K, Fujino M, et al. Nephritis-associated plasmin receptor and acute poststreptococcal glomerulonephritis: characterization of the antigen and associated immune response. $\mathbf{J}$ Am Soc Nephrol 15: 1785-1793, 2004.

2. Yamakami K, Yoshizawa N, Wakabayashi K, Takeuchi A, Tadakuma T, Boyle MD. The potential role for nephritis- associated plasmin receptor in acute poststreptococcal glomerulonephritis. Methods 21: 185-197, 2000.

3. Oda T, Yamakami K, Omasu F, et al. Glomerular plasmin-like activity in relation to nephritis-associated plasmin receptor in acute poststreptococcal glomerulonephritis. J Am Soc Nephrol 16: 247254, 2005.

4. Kikuchi Y, Yoshizawa N, Oda T, Imakiire T, Suzuki S, Miura S. Streptococcal origin of a case of Henoch-Schoenlein purpura nephritis. Clin Nephrol 65: 124-128, 2006.

5. Suga K, Kondo S, Matsuura S, et al. A case of dense deposit disease associated with a group A streptococcal infection without the involvement of $\mathrm{C} 3 \mathrm{NeF}$ or complement factor $\mathrm{H}$ deficiency. Pediatr Nephrol 25: 1547-1550, 2010.

6. Sawanobori E, Umino A, Kanai H, et al. A prolonged course of Group A streptococcus-associated nephritis: a mild case of dense deposit disease (DDD)? Clin Nephrol 71: 703-707, 2009.

7. Okabe M, Tsuboi N, Yokoo T, Miyazaki Y, Utsunomiya Y, Hosoya T. A case of idiopathic membranoproliferative glomerulonephritis with a transient glomerular deposition of nephritisassociated plasmin receptor antigen. Clin Exp Nephrol 16: 337341, 2012.

8. Uchida T, Oda T, Watanabe A, et al. Clinical and histologic resolution of poststreptococcal glomerulonephritis with large subendothelial deposits and kidney failure. Am J Kidney Dis 58: 113-117, 2011.

9. Vernon KA, Goicoechea de Jorge E, Hall AE, et al. Acute presentation and persistent glomerulonephritis following streptococcal infection in a patient with heterozygous complement factor $\mathrm{H}$ related protein 5 deficiency. Am J Kidney Dis 60: 121-125, 2012.

10. Oda T, Yoshizawa N, Yamakami K, et al. The role of nephritisassociated plasmin receptor (NAPlr) in glomerulonephritis associated with streptococcal infection. J Biomed Biotechnol 2012: 417675, 2012.

11. Fish AJ, Herdman RC, Michael AF, Pickering RJ, Good RA. Epidemic acute glomerulonephritis associated with type 49 streptococcal pyoderma. II. Correlative study of light, immunofluorescent and electron microscopic findings. Am J Med 48: 28-39, 1970.

12. Prasto J, Kaplan BS, Russo P, Chan E, Smith RJ, Meyers KE. Streptococcal infection as possible trigger for dense deposit disease (C3 glomerulopathy). Eur J Pediatr 173: 767-772, 2014.

13. Rodriguez-Iturbe B, Batsford S. Pathogenesis of poststreptococcal glomerulonephritis a century after Clemens von Pirquet. Kidney Int 71: 1094-1104, 2007.

(C) 2016 The Japanese Society of Internal Medicine http://www.naika.or.jp/imonline/index.html 